Christian Grobe

\title{
Die Lücke, die der empiristische rational choice-Ansatz lässt
}

Eine Replik auf Johannes Marx

Brückenschläge zwischen rationalistisch und konstruktivistisch inspirierten Theorien der Internationalen Beziehungen stehen seit Jahren im Zentrum der Disziplin. Dieser Forumsbeitrag setzt sich kritisch mit dem jüngsten Versuch in dieser intellektuellen Tradition auseinander. Anhand neuester Arbeiten aus dem Kreis der experimentellen Ökonomik zeigt er, warum das Vorhaben von Johannes Marx, den allgemeinen theoretischen Kern der Internationalen Beziehungen mit dem Kern von rational choice gleichzusetzen, von einer unüberwindbaren Hypothek belastet wird. Die für seinen Integrationsvorschlag notwendige Öffnung des rational choice-Ansatzes, vor allem für Prozesse des endogenen Präferenzwandels, verspielt nämlich nicht nur die Erklärungskraft dieses Ansatzes, sondern überdehnt ihn darüber hinaus konzeptionell. Zur Vermeidung dieser unerwünschten Nebeneffekte skizziert der vorliegende Beitrag eine alternative Konzeption von rational choice, die das essentielle theoretische Postulat fixer Präferenzen unangetastet lässt, aber dennoch eine integrative Perspektive auf die handlungstheoretische Debatte zwischen Konstruktivisten und Rationalisten eröffnet.

»Empirie und nicht Empirismus! «1 Wilhelm Dilthey

\section{Einleitung ${ }^{2}$}

Als der Konstruktivismus Mitte der 1990er Jahre zu einem alternativen metatheoretischen Fixpunkt neben dem Paradigma rationalen Handelns (rational choice) avancierte, wurde die Theorienlandschaft der Internationalen Beziehungen (IB) schlagartig unübersichtlicher. Zu der Diskussion über die relative Erklärungskraft verschiedener Theorievarianten innerhalb des rationalistischen Forschungsprogramms (Baldwin 1993), gesellte sich bald eine Paradigmen übergreifende Konversation zwischen rational choice-Vertretern und Konstruktivisten (Fearon/Wendt 2003; Katzenstein et al. 1998). In dieser Konversation haben Autoren aus beiden Lagern versucht, die neue

1 Dilthey hat dieses Motto in seiner Auseinandersetzung mit dem wissenschaftstheoretischen Empirismus geprägt (vgl. dazu das Vorwort des Herausgebers in Diltheys (1924: LXXVI) Gesammelte Schriften).

2 Für hilfreiche Kommentare zu früheren Versionen dieses Artikels danke ich der ZIBRedaktion und ihren Gutachterinnen und Gutachtern. 
theoretische Vielstimmigkeit zu einem harmonischen Chor zusammenzufügen. ${ }^{3}$ Und wo dies aufgrund unvereinbarer wissenschaftsphilosophischer Positionierungen unmöglich war, sollten wenigstens die jeweiligen Anwendungsdomänen dieser verschiedenen Theorien näher spezifiziert werden (ausführlich hierzu Checkel 2010).

In dieser bis heute sehr lebhaften intellektuellen Tradition steht auch der Beitrag von Johannes Marx (2010), der in der vorletzten Ausgabe dieser Zeitschrift erschienen ist. Unter dem Titel Is There a Hard Core of IR? schlägt Marx eine Rekonstruktion zentraler IB-Theorien in den Kategorien von rational choice vor. Erklärtes Ziel dieses Unternehmens ist es, die rationalistische Trias aus Neorealismus, Neoinstitutionalismus und Liberalismus mit dem Sozialkonstruktivismus Wendtscher Prägung zu versöhnen und alle vier Theorien »als kontextualisierte Spezialisierungen eines allgemeinen Modells der Internationalen Beziehungen« auszuweisen (Marx 2010: 41).

Zur Realisierung dieses Unternehmens interpretiert Marx den rationalistischen Ansatz in der empiristischen Tradition der Soziologie. In dieser Tradition werden die Präferenzen der Akteure nicht - wie sonst üblich - analytisch postuliert, sondern für einen jeden Akteur und eine jede Situation empirisch erhoben. ${ }^{4}$ Dies erlaubt einerseits die Berücksichtigung von vielfältigen sozialen Motiven in der Nutzenfunktion und andererseits den Wandel dieser Motive auch während der Interaktion. Diese weitgehende Öffnung der konzeptionellen Grundlagen ist nötig, um Wendts (1999: Kap. 3 und 4) Fokus auf konstitutive Ideen und reflexives Lernen in der Terminologie des Rationalismus rekonstruieren zu können. Doch anders als Marx glaubt, verspielt diese Öffnung die Erklärungskraft des rationalistischen Ansatzes und überdehnt ihn darüber hinaus konzeptionell. Damit lastet er seinem Vorhaben, den allgemeinen theoretischen Kern der IB mit dem Kern von rational choice gleichzusetzen, eine unüberwindbare Hypothek auf. ${ }^{5}$

Im Folgenden möchte ich diese Behauptung im Rahmen einer allgemeinen Diskussion zum aktuellen theoretischen Status von rational choice begründen. Dazu skizziere ich im zweiten Abschnitt kurz die beiden intellektuellen Traditionen des rationalistischen Ansatzes - die empiristische und die analytische. Diese Skizze bereitet den Boden, um im dritten Abschnitt zuerst die Aporien von Marx' empiristischer Rationalismuskonzeption offen zu legen und anschließend die Überlegenheit des

3 Auch die hiesige Kontroverse zwischen Rationalisten und Konstruktivisten, besser bekannt als ZIB-Debatte, segelt unter dieser Flagge. Für eine aktuelle Zusammenfassung der zahlreichen Beiträge zu dieser Debatte samt einer Darstellung ihrer inhaltlichen Verästelungen siehe Schäfer (2007).

4 Das analytische Verfahren gehört zum Standardvorgehen in allen bekannten rationalistischen IB-Theorien. So postuliert beispielsweise der neoliberale Institutionalismus, dass Staaten primär nach der Maximierung ihrer individuellen Wohlfahrt streben, um anschließend vor diesem Hintergrund die rege internationale Kooperation in einem interdependenten Selbsthilfesystem zu erklären. Analoge axiomatische Festlegungen sind auch im Neorealismus und der liberalen Theorieschule zu finden, wie bei Marx (2010: 48-57) selbst nachzulesen ist.

5 Marx (2010: 70) scheint sich dieser Gefahr bewusst zu sein, wenn er im Schlusswort schematisch auf alternative Bestimmungsmöglichkeiten des Kerns von rational choice verweist, die sein Rekonstruktionsvorhaben gefährden könnten. Leider erkundet er diese Alternativen nicht weiter, sondern beeilt sich, diese aus methodologischer Perspektive seinem Rationalismusverständnis für unterlegen zu erklären. 
analytischen Zugangs anhand der neuesten Erkenntnisse der experimentellen Ökonomik zu demonstrieren. Diese Überlegenheit, so das zentrale Argument, speist sich aus der erfreulichen Tatsache, dass der analytische Rationalismus - anders als sein empiristischer Gegenpart - erstmals die Formulierung empirisch gehaltvoller Theorien sozialer Präferenzen ermöglicht hat. Daher auch das einleitende Motto: »Empirie und nicht Empirismus! «. Der vierte Abschnitt befasst sich sodann mit der Frage nach dem Wandel von Präferenzen, die seit Mitte der 1990er Jahre die handlungstheoretische Debatte zwischen Konstruktivisten und Rationalisten prägt. ${ }^{6}$ Dabei wird die konstruktivistische Position untermauert, dass sich Prozesse des Präferenzwandels nicht gehaltvoll mit dem Instrumentarium von rational choice analysieren lassen. Zugleich wird aber auch deutlich, dass moderne rationalistische Ansätze im Geist der experimentellen Ökonomik nicht vor den vielfach nachgewiesenen Positionswechseln einzelner Akteure während einer Interaktion kapitulieren müssen, sondern entgegen der landläufigen konstruktivistischen Meinung diesen Wandlungsprozess auch bei Unterstellung fixer Präferenzen theoretisch erfassen können. An dieser Stelle weist der vorliegende Kommentar bereits über eine methodologische Kritik an Marx hinaus und eröffnet eine neue Perspektive auf die Diskussion zwischen Rationalisten und Konstruktivisten, wie der abschließende fünfte Abschnitt argumentiert - und zwar in einem Bereich, der schon seit langem von großem Interesse innerhalb der deutschsprachigen IB ist: der Theorie multilateraler Verhandlungen.

\section{Zwei intellektuelle Traditionen des rationalistischen Ansatzes}

Die Frage nach der Rolle rationalen Handelns in der Erklärung von Akteursinteraktionen ist ein Dauerbrenner des politikwissenschaftlichen Diskurses. Was dabei genau rationales Handeln konstituiert, ist allerdings bis heute umstritten und dementsprechend Inhalt zyklisch wiederkehrender Debatten (z.B. Friedman 1996; Green/Shapiro 1994; Hindmoor 2010). In ihrer engsten, traditionellen Fassung ruht die Theorie rationalen Handelns auf drei Pfeilern, die gemeinsam das altbekannte Erklärungsmodell des Homo oeconomicus tragen: Der Rationalitätsannahme, dem Eigennutzaxiom und der Unterstellung vollständiger Information. Die Rationalitätsannahme besagt, dass Individuen mit ihren Handlungen immer die beste Alternative aus allen ihnen zur Verfügung stehenden Optionen auswählen - oft als Maximierung unter Restriktionen abgekürzt. Das Objekt dieser Maximierung sind die Präferenzen der Individuen, die in einer widerspruchsfreien Ordnung zueinander stehen müssen. Die Rationalitätsannahme bildet den konstitutiven Bestandteil von rational choice - und damit einen unaufgebbaren harten Kern, wie auch Marx (2010: 47) annimmt. Das Eigennutzaxiom, das soziale Präferenzen wie etwa Fairness oder nicht-strategische Reziprozität aus der Präferenzordnung ausschließt, ist dagegen lediglich eine Zusatzannahme. Gleiches gilt für die Unterstellung vollständiger Information.

6 Stellvertretend seien hier in chronologischer Reihenfolge genannt: Risse-Kappen (1995); Keck (1997); Checkel (2001); Deitelhoff (2009); Grobe (2010); Risse/Kleine (2010). 
Die Preisgabe dieser beiden Zusatzannahmen zur Befreiung des rationalistischen Ansatzes aus seiner materiellen und hyperrationalen Engführung wurde vielfach diskutiert - allerdings mit unterschiedlichem Ausgang. Spätestens seit dem Aufkommen der Informationsökonomik Anfang der 1970er Jahre gehen nur noch die wenigsten rationalistischen Arbeiten von vollständig informierten Akteuren aus (vgl. Riley 2001). Das Eigennutzaxiom ist dagegen lange mit der Rationalitätsannahme verschmolzen geblieben (Camerer/Fehr 2006: 52, Fn. 3). Um dies zu ändern, haben sich über die letzten Jahrzehnte zwei unabhängige Diskussionsstränge entfaltet: Einer in der stark empirisch orientierten Forschungstradition der Soziologie, ein anderer im Geist der analytisch verfahrenden Ökonomik. Beide Diskussionsstränge teilen das Ziel, soziale Motive gehaltvoll in den rationalistischen Ansatz zu integrieren; an dieser Stelle enden aber auch schon die Gemeinsamkeiten.

Marx (2010) ist, wie seine Literaturauswahl unschwer erkennen lässt, eindeutig der soziologischen Denkschule um James Coleman, Hartmut Esser und Karl-Dieter Opp zuzuordnen. Referenzpunkt dieser Schule ist Max Webers Ansatz des verstehenden Erklärens mit seinen bekannten drei Logiken - der Situation, der Selektion und der Aggregation. ${ }^{7}$ Folglich greift auch Marx zur Explikation seines Verständnisses von rational choice auf diese drei Logiken zurück, konzentriert sich dabei aber weitestgehend auf die ersten beiden: Den handlungstheoretischen Selektionsmechanismus, an dem die Akteure ihr Verhalten ausrichten, definiert er minimalistisch über die Rationalitätsannahme. Die Logik der Situation, d.h. welche Motive ein Individuum seinem Handeln zugrunde legt und wie es seine Handlungsoptionen in der gegebenen Situation bewertet, soll dagegen im Detail empirisch erschlossen werden. Von der engen Fassung des traditionellen rationalistischen Ansatzes lässt Marx also nicht mehr übrig als einen inhaltsleeren Maximierungsmechanismus, der von Situation zu Situation, von Individuum zu Individuum spezifiziert werden muss.

Ganz anders dagegen die Versuche innerhalb der Ökonomik - konkret: der experimentellen Ökonomik -, die Marx in seinem Beitrag leider gänzlich unerwähnt lässt. Auch sie zielen auf die Integration sozialer Motive in den rationalistischen Ansatz ab, jedoch ohne dabei auf empirische Brückenhypothesen zurückzugreifen, die das individuelle Handlungskalkül der Akteure jeweils aus der spezifischen Situation herleiten. Vielmehr basieren auch die in dieser neuen Forschungstradition entwickelten Modelle auf einer analytischen Zuschreibung von Erwartungen, Bewertungen und Motiven - genau wie traditionelle Varianten von rational choice. Dennoch haben sie einen erheblichen Beitrag zu einem verbesserten Verständnis altruistischen Handelns in sozialen Interaktionen geleistet (für einen Überblick siehe Falk/Heckman 2009; Ostrom 2006).

Marx (2010: 70) urteilt daher vorschnell, wenn er in seinem Schlusswort die Überlegenheit seines empiristischen Verständnisses von rational choice gegenüber einer

7 Eine ausführliche Darstellung dieser drei Logiken mit weiteren Literaturhinweisen zu ihrer Geschichte findet sich bei Esser (1993: 91-102). Die Originalüberlegungen lassen sich auf den eröffnenden Seiten von Wirtschaft und Gesellschaft nachlesen (Weber 1972: 1-9). Eine knappe Einordnung in die Forschungslogik der Sozialwissenschaften bietet Hollis (2002: 147-151). 
analytischen Rationalismuskonzeption suggeriert. ${ }^{8}$ Recht hat er allerdings, wenn er an gleicher Stelle betont, dass die relative wissenschaftstheoretische Attraktivität unterschiedlicher Varianten des rationalistischen Ansatzes allein über methodologische Argumente zu bestimmen sei. Beschränkt man sich dabei nicht nur, wie Marx fordert, auf das wenig wertvolle Quantitätskriterium der Anwendungsbreite, sondern fokussiert sich auf die tatsächliche Erklärungskraft - sprich: den empirischen Gehalt - der beiden Alternativen, zeigt sich die Überlegenheit der analytischen Tradition, wie der nächste Abschnitt erläutert.

\section{Empiristischer und analytischer Rationalismus - einige Anmerkungen zur relativen Erklärungskraft}

Im Rahmen dieses Abschnitts möchte ich zuerst einen Blick auf die Arbeiten des Soziologen Karl-Dieter Opp werfen, auf die sich Marx (2010) in seinem Beitrag vielfach stützt (Abschnitt 3.1). Nachdem dieser Blick die Schwachstellen des empiristischen Verständnisses von rational choice offenbart hat, präsentiere ich eine alternative Rationalismuskonzeption in der Tradition der analytisch verfahrenden Ökonomik (Abschnitt 3.2). Dabei wird deutlich, dass diese Konzeption einen höheren Erklärungsgehalt aufweist als ihr empiristischer Gegenpart.

\subsection{Aporien empiristischer Konzeptionen rationalen Handelns}

Karl-Dieter Opp gebührt ein großes Verdienst: In einer Zeit, in der rationales Handeln gleichbedeutend mit dem Handeln des Homo oеconomicus war, hat er erfolgreich für ein neues Rationalismusverständnis mit Fokus auf soziale Präferenzen und subjektive Situationsdeutungen gestritten; allerdings ohne damit den Erklärungsgehalt des rationalistischen Ansatzes signifikant zu erhöhen, wie ein Blick auf zwei seiner prominenteren Arbeiten offenbart: Soft Incentives and Collective Action: Participation in the Anti-Nuclear-Movement (Opp 1986) sowie Rational Choice and Collective Action (Muller/Opp 1986). ${ }^{9}$

8 In seiner im Jahr 2006 erschienenen Monografie Vielfalt oder Einheit der Theorien in den Internationalen Beziehungen, in der Marx seinen hier diskutierten Rekonstruktionsversuch das erste Mal vorgestellt hat, kommt dieser Überlegenheitsanspruch noch klarer zum Ausdruck (Marx 2006: 110-129). Auf den entsprechenden Seiten wird aber bereits deutlich, dass die Quelle seines Urteils eine Fehleinschätzung ist. Denn er setzt den analytischen Zugang, unter Verweis auf die Arbeiten von Anthony Downs und Mancur Olson, mit dem Homo oeconomicus gleich und übersieht damit die vielfältigen Neuerungen der experimentellen Ökonomik.

9 Die hier vorgetragene Kritik richtet sich nur gegen einen kleinen Teil von Opps umfänglichem Werk (einen Überblick bietet die Bibliographie in Diekmann et al. 2008: 335-340). Auch schmälert sie weder sein Verdienst, ein Bewusstsein für die Limitationen des Homo oeconomicus-Modells in Politikwissenschaft und Soziologie geweckt zu haben noch seine Leistungen in zahlreichen anderen Forschungsgebieten. 
In beiden Arbeiten, die in führenden politikwissenschaftlichen Fachzeitschriften erschienen sind, unternimmt Opp den Versuch, das Engagement in der deutschen Anti-Atomkraft-Bewegung unter Rückgriff auf die empiristische Variante von rational choice zu erklären. Dazu hat er mit seinem Team rund 400 Atomkraftgegner im Raum Hamburg interviewt, um sie unter anderem bezüglich ihrer Bewertung atomarer Energie, ihrer subjektiven Einschätzung eines möglichen Protesterfolgs, den normativen Verhaltenserwartungen in ihrem Umfeld und ihrer allgemeinen Protesthaltung zu befragen. Mit den Antworten auf diese Fragen wurde anschließend ein Set aus neun unabhängigen Variablen konstruiert und deren relativer Erklärungsbeitrag zum beobachteten Protestverhalten in einer multivariaten statistischen Analyse untersucht. ${ }^{10}$ Das Ergebnis dieser Untersuchung offenbart ein eindeutiges Muster: Zu engagierten Aktivisten werden diejenigen Atomkraftgegner, die

- $\quad$ starke Ängste gegenüber der Atomenergienutzung hegen und davon ausgehen, dass ihr Engagement zu einer signifikanten Reduktion von Atomkraftwerken führt;

- den Protest gegen die Atomenergie als ihre moralische Pflicht ansehen oder einfach pure Unterhaltung aus dieser Aktion ziehen;

- $\quad$ sich in einer Subkultur bewegen, die Teile ihres Selbstverständnisses aus dem Protest gegen die Atomkraft zieht und daher das Ansehen in der relevanten peer group vom Engagement in der Anti-Atomkraft-Bewegung abhängig macht.

Für Akteure mit diesen Motiven und Einschätzungen ist der erwartete Nutzen aus einem Engagement gegen die Atomkraft hoch genug, um die klassische Dilemmastruktur kollektiven Handelns (Olson 1965: Kap. 1) auszuhebeln, wodurch das weitläufige Protestverhalten als Resultat eines rationalen Maximierungskalküls erklärbar wird. ${ }^{11}$ Doch was lernen wir aus dieser Erklärung? Nicht sonderlich viel, möchte ich behaupten - und dies aus zwei Gründen.

Erstens drängt sich schon bei oberflächlicher Betrachtung der Ergebnisse ein Trivialitätsverdacht auf. Denn es wird lediglich bestätigt, was aufmerksame Beobachter längst wussten: Je stärker die negative Einstellung erklärter Atomkraftgegner gegenüber der Kernenergie, desto ernsthafter ihr Engagement gegen die weitere Nutzung dieser Energieform. Zwar gelingt Opp und seinen Mitarbeiter mit ihrer survey-Analyse eine wertvolle statistische Deskription des ideologischen Milieus der AntiAtomkraft-Bewegung samt den vielfältigen persönlichen Einstellungen ihrer Mitglieder. Dieses »ideologische Syndrom«, wie es Opp (1986: 106) bezeichnet, lehrt uns jedoch nichts über die kausalen Pfade, die einzelne individuelle Motive und Einschätzungen mit dem beobachteten Protestverhalten verbinden. Ob dieses Verhalten also tatsächlich das Resultat eines maximierten Erwartungsnutzens ist oder doch

10 Für ausführliche Informationen zum Forschungsdesign siehe Opp (1986: 106-112).

11 Ich setze an dieser Stelle voraus, dass die berichteten Ergebnisse gängigen Validitätsansprüchen genügen, was keineswegs unmittelbar klar ist. So impliziert beispielsweise die alleinige Konzentration auf Atomkraftgegner eine erhebliche Verzerrungsgefahr durch Selektionseffekte. Besonders schwerwiegend dabei ist, dass durch diese Sample-Auswahl eine wesentliche Erklärungsvariable konstant gehalten wurde: die Wohnnähe zu einem Atomkraftwerk. 
gänzlich andere Ursachen hat, ist auf Basis der gelieferten Korrelationen nicht zu ermitteln. Solange die Logik der Selektion als inhaltsleere Maximierungsregel spezifiziert wird, bleibt das individuelle Handlungskalkül eine black box mit eingeschränkter Erklärungskraft. ${ }^{12}$

Diese theoretische Unterdeterminiertheit führt bereits über den Trivialitätsverdacht hinaus und deutet, zweitens, auf die wesentliche Schwäche des empiristischen Ansatzes: Dort, wo die individuellen Handlungskalküle immer erst situativ erhoben werden, lauert die ständige Gefahr, neben allgemeinen Verhaltensdeterminanten auch zahlreiche arbiträre Faktoren in das Erklärungsmodell einfließen zu lassen - in der Statistik als Problem des overfitting bekannt (vgl. Elster 2007: 48; Kennedy 2008: 357). Zur Vermeidung dieses verzerrenden Effekts wäre es zwingend notwendig, vom spezifischen Kontext der Untersuchung zu abstrahieren, um anschließend die vielfältigen, unverbundenen Motive und Einschätzungen der Akteure in eine allgemeine Nutzenfunktion zu überführen und auf dieser Basis überprüfbare Implikationen für das Protestverhalten in anderen Regionen/Akteurskollektiven zu generieren. Nur so ist der Schritt von einer deskriptiv aufklärenden Momentaufnahme zu einer verallgemeinerbaren und empirisch gehaltvollen Erklärung des Protestkalküls innerhalb der deutschen Anti-Atomkraft-Bewegung möglich. Diesen Schritt zu gehen bedeutete allerdings, von einer rein empiristischen Rationalismuskonzeption Abstand zu nehmen und sich der analytischen Tradition zu öffnen. ${ }^{13}$

\subsection{Analytischer Rationalismus und die Entwicklung empirisch gehaltvoller Theorien sozialer Präferenzen}

Um gleich vorab ein weitläufiges Missverständnis auszuräumen: Arbeiten in der analytischen Tradition von rational choice entwickeln ihre Erklärungen nicht frei von empirischen Einsichten. Zwar wird das individuelle Handlungskalkül nicht für einen jeden Akteur situationsspezifisch erhoben, sondern als zentraler Inhalt der Nutzenfunktion axiomatisch festgelegt. Dies geschieht jedoch auf Basis zahlreicher empirischer Befunde, wie die neuesten Arbeiten aus dem Kreis der experimentellen Ökonomik belegen. Hier ist leider nicht der Ort, den Einfluss dieser Forschung auf den rationalistischen Ansatz, die mittlerweile ganze Monografien und Handbücher füllt (Camerer 2003; Plott/Smith 2008), zu detaillieren. Vielmehr konzentriere ich mich

12 Opp (1999: 181-188) hat bereits in einer früheren Schrift versucht, diesen nahe liegenden Trivialitätsverdacht zu zerstreuen. Dabei hat er allerdings eine wenig erfolgreiche negative Verteidigungsstrategie gewählt: Anstatt den Vorwurf der Trivialität inhaltlich zu entkräften, hat er lediglich gezeigt, dass derselbe Vorwurf auch gegen inhaltlich stärker spezifizierte Rationalismuskonzeptionen gerichtet werden kann. So wahr diese Feststellung ist, so wenig kann sie die berechtigte Kritik an seinem empiristischen Rationalismus widerlegen.

13 Interessanterweise ist dieser Schritt in Mullers und Opps Rational Choice and Rebellious Action bereits implizit angelegt. So postulieren die beiden Autoren zu Beginn dieses Beitrags eine allgemeine Nutzenfunktion (Muller/Opp 1986: 474), die ohne weiteres mit ihren späteren empirischen Parameterschätzungen gefüllt werden könnte, um damit die Erklärungskraft ihres Ansatzes an anderen Protestregionen und Akteuren zu testen. 
im Folgenden auf einen kleinen Ausschnitt dieser Literatur, um den überlegenen Erklärungsbeitrag der analytischen Rationalismuskonzeption zu belegen. Der Fokus wird dabei auf aktuellen Erkenntnissen aus experimentellen Diktatorspielen liegen.

In der einfachsten Version dieses Spiels erhält ein Individuum (der Diktator) einen Geldbetrag, den er beliebig zwischen sich und einem anderen Akteur (dem Empfänger) aufteilen kann. Diktatorspiele sind damit Nullsummenspiele, mit denen die soziale Motivation der Akteure frei von verzerrenden strategischen Einflüssen untersucht werden kann.

Gleich die ersten Diktatorexperimente Mitte der 1990er Jahre haben die Vertreter des traditionellen rationalistischen Ansatzes in große Erklärungsnöte gebracht. Denn anstatt ihren materiellen Eigennutzen zu maximieren, wie vom Modell des Homo oeconomicus prognostiziert, gaben Diktatoren einen signifikanten Anteil an die Empfänger $\mathrm{ab}$ - durchschnittlich 20\% des Ausgangsbetrags (Forsythe et al. 1994: 357). In späteren Replikationen hat sich diese Abgabequote sogar nochmals leicht erhöht und dann stabil bei 20-30\% eingependelt (Camerer 2003: 57-58). Die natürliche Antwort auf diese Anomalie war es, den Standardansatz schlicht um soziale Präferenzen - wie beispielsweise Ungleichheitsaversion - zu ergänzen (Fehr/Schmidt 1999; Bolton/Ockenfels 2000). Diese Strategie setzte im Wesentlichen die alte Forderung um, ein Streben nach fairer Verteilung materieller Güter gleichberechtigt neben der Maximierung der eigenen monetären Auszahlung in der klassischen Nutzenfunktion abzubilden und damit altruistisches Handeln konzeptionell in den rationalistischen Ansatz zu integrieren.

Doch neue Experimente, die im Anschluss an die Entwicklung dieser ergebnisbasierten Theorien sozialer Präferenzen zu ihrem Test durchgeführt wurden, haben dieser Hoffnung ein schnelles Ende bereitet. Anstatt in Spielen mit leicht verändertem Kontext immer wieder dieselbe Verteilungsentscheidung zu treffen, variierte das Verhalten der Experimentteilnehmer merklich. So zogen es beispielsweise viele Individuen vor, einen fixen Betrag ausgezahlt zu bekommen anstatt an einem Diktatorspiel teilzunehmen, in dem sie über eine höhere Ausstattung als diesen Fixbetrag hätten verfügen können - vorausgesetzt der mögliche Spielpartner erfährt nichts von dieser exit-Entscheidung (Dana et al. 2006: 197-199). Ein ähnlicher self serving bias war oft auch dann zu beobachten, wenn die Spieler die Konsequenzen ihrer Entscheidungen nicht mit vollkommener Sicherheit kannten. In solchen Fällen neigten sie dazu, die Wahrscheinlichkeit für eine unfaire Behandlung ihres Spielpartners drastisch zu unterschätzen, um so die Erhöhung ihres eigenen Profits zu rechtfertigen (Dana et al. 2007: 74-77; Haisley/Weber 2010: 619-622).

Rein ergebnisbasierte Theorien sozialer Präferenzen mit ihrem Fokus auf kontextunabhängige Fairnessmotive müssen vor diesem Verhalten kapitulieren. Wären die Akteure nämlich tatsächlich nur an der finalen Verteilung des Ausgangsbetrags interessiert, sollten sie unabhängig vom Transparenzgrad der Entscheidungssituation immer die gleiche Allokation wählen und höhere Anfangsausstattungen gegenüber niedrigeren bevorzugen. Die Neigung, sich erst unter dem Schleier der Anonymität oder bei geringer direkter Verantwortung für das Verteilungsergebnis in den Profit maximierenden Homo oeconomicus zu verwandeln, deutet daher auf ein Interesse 
dieser Akteure an einer positiven Außenwahrnehmung, das faires Verhalten nur dann gebietet, wenn es dem eigenen Image hilft.

Entsprechend diesen empirischen Befunden haben James Andreoni und Douglas Bernheim (2009) jüngst eine Theorie des sozialen Images vorgeschlagen, deren Kern eine Nutzenfunktion bildet, die das individuelle Handlungskalkül im Spannungsfeld zwischen egoistischer Profitmaximierung, altruistischen Fairnessbestrebungen und einer Sorge um die eigene Außenwahrnehmung modelliert. In dieser Spezifikation der Nutzenfunktion sind - anders als in älteren, ergebnisbasierten Theorien - neben Präferenzen über die finale Verteilung auch Präferenzen über die Vorstellungen anderer Akteure relevant für die Abgabeentscheidung. Denn einem auf sein Image fokussierten Diktator ist es nicht wichtig, wirklich fair zu sein; er möchte lediglich von anderen als fair wahrgenommen werden.

Für Akteure mit dieser Nutzenfunktion können nicht nur die experimentell beobachteten exit-Entscheidungen ex post erklärt, sondern noch weitere beobachtbare Implikationen abgeleitet werden: Neben dem bekannten Anteil egoistischer Diktatoren (Abgabequote: 0\%) und fairer Diktatoren (Abgabequote: 50\%), wird es zahlreiche Diktatoren geben, deren Abgabeverhalten mit dem Transparenzgrad ihrer Entscheidung variiert.

Um diese Implikation zu testen, haben Andreoni und Bernheim ein neues Diktatorexperiment durchgeführt - bestehend aus vier treatments, in denen die Diktatoren jeweils über einen Ausgangsbetrag in Höhe von 20 Dollar verfügen konnten. Ihr erstes treatment glich der klassischen Variante des Diktatorspiels, wie sie weiter oben beschrieben wurde. Die drei weiteren haben sukzessive die Transparenz der Diktatorentscheidung reduziert. Dazu wurde die finale Allokation mit steigenden Wahrscheinlichkeiten von 25, 50 und 75\% nicht mehr vom Diktator frei bestimmt, sondern von den beiden Versuchleitern auf 19 Dollar für den Diktator und einen Dollar für den Empfänger festgesetzt. ${ }^{14}$ Durch diesen Mechanismus war es für die Empfänger immer weniger zu ermitteln, ob der ihnen zugewiesene Betrag auf der intentionalen Abgabeentscheidung des Diktators oder auf der Festsetzung der Versuchsleiter beruhte.

Die Ergebnisse dieses Experiments sprechen eindeutig für die Erklärungskraft der vorgeschlagenen Image-Theorie: Diktatoren, die um ihr Fairness-Image bemüht sind, entscheiden sich bei vollkommener Transparenz für eine 50-50-Aufteilung des verfügbaren Betrags und rücken mit steigender Wahrscheinlichkeit einer Festsetzung des Abgabebetrags auf einen Dollar von dieser Strategie ab. Kann ihre Verteilungsentscheidung nämlich nicht von den Empfängern mit Sicherheit auf sie zurückgeführt werden, müssen sie keinen Verlust ihres sozialen Images befürchten, wenn sie ihren materiellen Eigennutzen mehren. Dementsprechend sinkt die Quote der fairen 50-50-

14 Zur Illustration: Entscheidet sich ein Diktator im letzten treatment, die Hälfte des verfügbaren Betrags (zehn Dollar) an den ihm zugewiesenen Empfänger abzugeben, wird diese intentional gewählte 50-50-Allokation nur mit einer Wahrscheinlichkeit von $25 \%$ erreicht. Mit der Komplementärwahrscheinlichkeit von 75\% erhält der Empfänger dagegen lediglich einen Dollar und die Diktatorentscheidung bleibt ohne Einfluss auf das tatsächliche Verteilungsergebnis. 
Verteilung von treatment zu treatment, wohingegen sich ein wachsender Pool für die Abgabe von einem Dollar entschließt (siehe Abbildung 1).

Abbildung 1: Abgabebeträge in einem experimentellen Diktatorspiel mit Image-Effekten

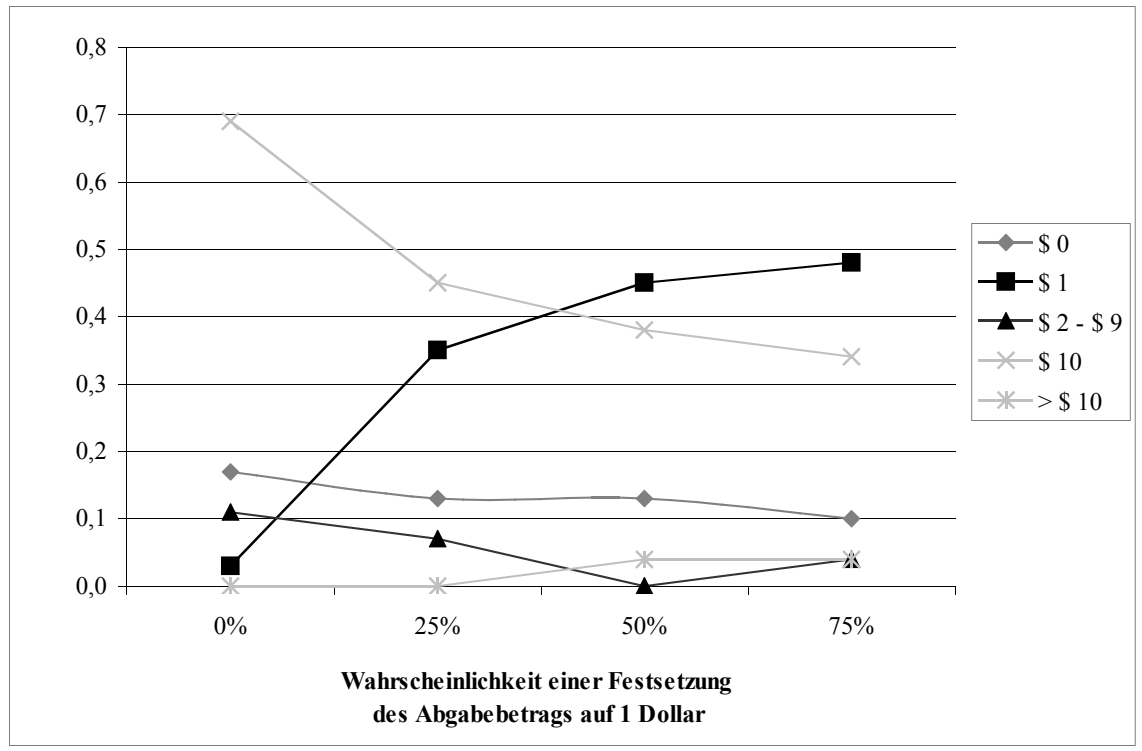

Quelle: Andreoni/Bernheim 2009: 1622.

Anders als die empiristisch inspirierten Studien von Opp reichen die analytischen Arbeiten experimenteller Ökonomen weit über die Feststellung bloßer Korrelationen zwischen erklärten individuellen Handlungsmotiven und beobachtetem Verhalten hinaus, indem sie die inhaltliche Maximierungsregel vorab spezifizieren. Damit stellen sie die fehlende kausale Verbindung zwischen situativen Rahmenbedingungen und tatsächlichem Handeln der Akteure her. Durch diese Spezifizierung wird die black box des Handlungskalküls zielführend geöffnet und eine Überprüfung der postulierten Zusammenhänge in zahlreichen Kontexten möglich - natürlich auch außerhalb des Labors. So macht die Image-Theorie beispielsweise eine klare Aussage zu den Erfolgschancen neuester Versuche einiger US-Bundesstaaten im Kampf gegen Steuerbetrug. Diese Versuche zielen darauf ab, die Steuermoral über eine Online-Veröffentlichung der Namen von Steuersündern mit dem größten Hinterziehungsvolumen 
zu erhöhen. ${ }^{15}$ Sollten potenzielle Steuersünder tatsächlich an ihrem sozialen Image interessiert sein, wird diese angedrohte Bloßstellung zu insgesamt weniger Steuerverbrechen führen.

Es sind diese vielfältigen beobachtbaren Implikationen, die für eine überlegene Erklärungskraft der analytischen Rationalismuskonzeption bürgen und erstmals die Formulierung empirisch gehaltvoller Theorien sozialer Präferenzen ermöglicht haben. ${ }^{16}$

\section{Irrungen und Wirrungen - einige Klarstellungen zur Frage des Präferenzwandels ${ }^{17}$}

Experimentelle Ökonomen haben zwar das Eigennutzaxiom des traditionellen rationalistischen Ansatzes verabschiedet und durch einen reichhaltigeren Präferenzbegriff ersetzt. Das alte Diktum der Präferenzstabilität über den Zeitraum der Interaktion (Becker 1978: 5; Kirchgässner 2008: 221) haben sie jedoch aufrechterhalten. Marx' empiristisches Verständnis höhlt also nicht nur die Erklärungskraft des rationalistischen Ansatzes aus, sondern überdehnt ihn noch dazu konzeptionell. Denn nach herrschender Auffassung verstößt ein endogener Wandel von Präferenzen eindeutig gegen das Postulat einer transitiven, widerspruchsfreien Nutzenfunktion und stellt damit eine Verletzung des harten Kerns von rational choice dar (Bardsley et al. 2010: 128-131). Nun ist Marx mit seinen Ansichten nicht allein, sondern zitiert durchaus namhafte Vertreter der ökonomischen Zunft, die ebenfalls aus rationalistischer Perspektive von endogenem Präferenzwandel sprechen.

Bei näherer Betrachtung der vier Studien, mit denen Marx (2010: 62, Fn. 17) seine Position zu stützen versucht, ist allerdings schnell zu erkennen, dass er einer weit verbreiteten Konzeptverwirrung durch unpräzisen Begriffsgebrauch ihrer Autoren erlegen ist: Einmal beziehen sich diese nämlich auf die Konstitution von Präferenzen im Rahmen von Sozialisationsprozessen (Ben-Ner/Putterman 2000; Bowles 1998) und ein anderes Mal diskutieren sie Verhaltensänderungen der Akteure in einem evolutionären spieltheoretischen Ansatz zur Erklärung kooperativen Verhaltens (Güth et al. 2000; Vogt 2000) - beides jeweils unter dem Stichwort »endogener Präferenzen«.

In den beiden Arbeiten mit Fokus auf die Sozialisation der Akteure ist sicherlich Raum für langfristigen Präferenzwandel über die verschiedenen Stadien der Ent-

15 Eine Liste mit den teilnehmenden Staaten inkl. der 250 größten Steuerhinterzieher findet sich auf der Homepage der Los Angeles Times (2011). Auch die britische Steuerbehörde plant die Veröffentlichung einer solchen shaming-Liste auf ihrer Homepage (HM Revenue \& Customs 2011).

16 Als Randnotiz: Vor diesem Hintergrund hat auch Harald Müllers (1995: 372) alte Einschätzung aus den Zeiten der ZIB-Debatte ihr Haltbarkeitsdatum überschritten: Die »Altruistin « ist heutzutage mehr »als eine Alibifigur, um die fatalen Einschränkungen zu verdecken, die den Akteursbegriff des Rational-Choice-Ansatzes in der praktischen Forschung kennzeichnen«.

17 Die Argumentation dieses Abschnitts baut auf früheren Arbeiten des Verfassers zu diesem Thema auf (Grobe 2010: 7-10). 
wicklung; doch hilft rational choice zum Verständnis dieses Prozesses nicht weiter, da er sich schwerlich in intentionalen Maximierungskalkülen fassen lässt. Die zwei Studien, die sich mit der Modellierung evolutionärer Kooperationsdynamiken befassen, setzen dagegen die beobachteten Verhaltensänderungen fälschlicherweise begrifflich mit einem Präferenzwandel gleich, obwohl sie eindeutig das Resultat einer revidierten Situationsdeutung sind: Während sich nämlich das Kooperationsspiel entfaltet - in dem Akteure mit sozialen und egoistischen Präferenzen aufeinandertreffen, Egoisten einen klaren Anreiz zur Defektion haben und nicht immer von den sozialen Typen unterscheidbar sind -, kann es sich für eigeninteressierte Akteure bei bestimmten Typenkonstellationen und Informationstechnologien durchaus lohnen, sich in den nächsten Runden kooperativ zu verhalten, um ihren eigenen Profit zu maximieren. Damit sterben zwar die egoistischen Strategien im Spielverlauf sukzessive aus, nicht aber die egoistischen Präferenzen. Der zentrale Treiber hinter dieser Entwicklung sind von Runde zu Runde aktualisierte Einschätzungen (beliefs) über die zu erwartende Auszahlung bei kooperativem Verhalten. Marx irrt also, wenn er behauptet, dass Präferenzwandel nichts anderes sei »als das Erlernen neuer Handlungsalternativen oder neuer Bewertungen dieser Handlungsalternativen « (Marx 2010: 62). Denn in den eben betrachteten Studien sind diese beiden Vorgänge das Resultat eines Einschätzungswandels und eben gerade nicht eines Präferenzwandels. ${ }^{18}$

Marx ist allerdings mit diesem Irrtum nicht allein. Vielmehr zieht dieser sich durch die gesamte IB-Literatur zum Thema Präferenzwandel, wie ein Blick auf neuere konstruktivistische Arbeiten zur Rolle argumentativer Überzeugung in multilateralen Verhandlungen offenbart (z.B. Checkel 2001; Risse/Kleine 2010). So definiert Jeffrey Checkel (2001: 562) einen Überzeugungsvorgang als einen »process of interaction that involves changing attitudes about cause and effect in the absence of overt coercion. It is thus a mechanism through which preference change may occur«. Ein Einschätzungswandel der Akteure wird in dieser Definition zur Voraussetzung für einen Präferenzwandel, obwohl er doch aus rationalistischer Perspektive bereits eine Alternative darstellt, um Verhaltensänderungen in Folge argumentativen Austauschs zu erklären.

Die problematischen Folgen dieser konzeptionellen Verwirrung lassen sich besonders gut an dem häufig gewählten Beispiel zum Einfluss argumentativer Rede in Gerichtsverhandlungen (Risse/Kleine 2010: 711) illustrieren: Natürlich muss der Richter gegenüber den Argumenten von Verteidigern und Staatsanwälten offen sein, sich in seinem Meinungsbildungsprozess gar vom besseren Argument überzeugen lassen. Dieser Prozess ist jedoch das schlichte Resultat einer schrittweisen Bewertung der Glaubwürdigkeit und Stichhaltigkeit vorgebrachter Evidenz. In rationalistischer Terminologie ausgedrückt, spielen die Akteure vor Gericht nichts anderes als ein proze-

18 Diese konzeptionelle Ungenauigkeit führt Marx (2010: 65) dazu, Formen des sozialen Lernens mit einem Wandel von Präferenzen gleichzusetzen. Das Erlernen von Wegen, »um möglichst ohne große Such- und Informationskosten den eigenen Nutzen zu maximieren« ist jedoch etwas gänzlich anderes als die Präferenzen zu ändern, die diesen Nutzen zuallererst konstituieren. Damit sind diese erlernten Wege nichts anderes als unterschiedliche Strategien, um fixe Präferenzen optimal zu realisieren. 
dural wohl definiertes Überzeugungsspiel (Glazer/Rubinstein 2004: 1717-1720), dessen Ausgang wesentlich über belief-basierte Überzeugungsvorgänge erklärt werden kann. Warum der Richter dagegen Präferenzen über den Verhandlungsausgang haben sollte, die er im Lichte vorgebrachter Argumente anpasst, ist nicht so leicht nachzuvollziehen. ${ }^{19}$

\section{Zusammenfassung und Ausblick}

Die Attraktivität und Erklärungskraft von rational choice beruht auf einfachen analytischen Annahmen über die Präferenzstruktur der Akteure, die empirisch gehaltvolle Aussagen über das individuelle Handeln in strategischen Entscheidungssituationen ermöglichen. Eine Abkehr von dieser analytischen Konzeption zugunsten eines empiristischen Rationalismusverständnisses verspielt diese Stärken: Zur Feststellung individueller situationsspezifischer Handlungskalküle, die sich stets durch einen komplexen Mix aus egoistischen und altruistischen Motiven auszeichnen, hat der rationalistische Ansatz genauso wenig beizutragen, wie zur Aufklärung reflexiver Lernprozesse, die einen Wandel der Präferenzstruktur voraussetzen. Vor diesem Hintergrund ist es unmöglich, Wendts Sozialkonstruktivismus als eine Theorievariante im rationalistischen Forschungsprogramm zu rekonstruieren, wie Marx es jüngst versucht hat.

Diese Betonung der konzeptionellen Gräben zwischen Rationalismus und Konstruktivismus soll jedoch nicht die vielfältigen Ansatzpunkte für Brückenschläge zwischen diesen beiden metatheoretischen Fixpunkten in der IB-Theorie verdecken: Trotz des Fokusses auf allgemeine, axiomatisch postulierte Präferenzen bleibt nämlich auch in der analytischen Rationalismuskonzeption genügend Raum für die Untersuchung sozialer Interaktionen, in denen die Akteure nicht allein von ihrem Eigeninteresse getrieben werden, sondern sich in einem Spannungsfeld zwischen egoistischen und altruistischen Motiven bewegen, wie ein Blick auf die neuesten Arbeiten aus dem Kreis experimenteller Ökonomen gezeigt hat. ${ }^{20}$ Auch das Bekenntnis zu fixen Präferenzen heißt nicht, dass der rationalistische Ansatz vor den vielfach beobachteten Verhaltensänderungen einiger Akteure während einer Interaktion kapitulieren müsste. So ist beispielsweise der nachgewiesene Einfluss argumentativer Rede auf die Positionen einzelner Staaten in internationalen Verhandlungen ohne weiteres über den Mechanismus belief-induzierter Verhaltensänderungen zu erklären. Konstruktivisten mussten diese Möglichkeit bisher jedoch übersehen und argumentativ

19 Belief-basierte Verhaltensänderungen in Reaktion auf sprachlichen Austausch sind dabei nicht auf faktische Argumente über Zustände der Welt beschränkt, sondern können auch durch normative Argumentationsvorgänge ausgelöst werden (vgl. Grobe 2009: 274-276).

20 An der Politikwissenschaft, insbesondere an der Theorie multilateraler Verhandlungen, ist diese Entwicklung bisher allerdings vollständig vorbeigegangen, so dass rational choice noch heute auf bargaining zwischen eigeninteressierten Akteuren reduziert wird (Morin/ Gold 2010: 566; Risse/Kleine 2010: 709) und die Möglichkeit einer Integration sozialer Präferenzen in den rationalistischen Ansatz weiterhin undenkbar erscheint (Sarigil 2010: 475-477). 
herbeigeführte Verhaltensänderungen immer mit einem Präferenzwandel gleichsetzen, da sie in ihren Arbeiten nicht sauber zwischen belief- und präferenzbasierten Erklärungen trennen und damit argumentativen Austausch in rationalistischen Ansätzen fälschlicherweise als bloßes Epiphänomen herrschender Macht- und Interessenkonstellationen abtun (zuletzt Deitelhoff 2009: 46; Risse/Kleine 2010: 708).

Am Ende ist es natürlich eine rein empirische Frage, welcher dieser beiden Mechanismen den Einfluss argumentativer Rede besser einfängt. Zu ihrer Beantwortung ist die jüngste Hinwendung konstruktivistischer Theoretiker zu den institutionellen Erfolgsbedingungen kommunikativen Handelns nur zu begrüßen. Denn dort, wo der Einfluss argumentativer Rede nicht mehr von der Interaktionseinstellung der Akteure abhängt, sondern von den Charakteristika des institutionellen Kontexts, kann sich eine neue Verbindungslinie zwischen rationalistischen und konstruktivistischen Verhandlungstheorien öffnen. Allerdings erst dann, wenn Vertreter beider Theorieschulen die kausalen Pfade zwischen dem Austausch von Argumenten und Positionswechseln näher spezifizieren, um anschließend belief- und präferenzinduzierte Verhaltensänderungen in Abhängigkeit variierender Kontextbedingungen unterscheiden zu können (vgl. Grobe 2010). Werden bei diesem Schritt dieselben Kontextbedingungen identifiziert, rückt der konzeptionelle Unterschied zwischen belief- und Präferenzwandel in den Hintergrund und der metatheoretische Graben zwischen Konstruktivisten und Rationalisten kann auf dem stabilen Boden empirischer Forschungsergebnisse überbaut werden. Damit wäre am Ende viel mehr gewonnen, als durch wechselseitige methodologische Assimilierungsversuche jemals zu erreichen sein wird.

\section{Literatur}

Andreoni, James/Bernheim, B. Douglas 2009: Social Image and the 50-50 Norm: A Theoretical and Experimental Analysis of Audience Effects, in: Econometrica 77: 5, 1607-1636.

Baldwin, David A. 1993: Neorealism and Neoliberalism: The Contemporary Debate, New York, NY.

Bardsley, Nicholas/Cubitt, Robin/Loomes, Graham/Moffat, Peter/Starmer, Chris/Sugden, Robert 2010: Experimental Economics: Rethinking the Rules, Princeton, NJ.

Becker, Gary S. 1978: The Economic Approach to Human Behavior, Chicago, IL.

Ben-Ner, Avner/Putterman, Louis 2000: On Some Implications of Evolutionary Psychology for the Study of Preferences and Institutions, in: Journal of Economic Behavior \& Organization 43: 1, 91-99.

Bolton, Gary E./Ockenfels, Axel 2000: ERC-A Theory of Equity, Reciprocity, and Competition, in: American Economic Review 90: 1, 166-193.

Bowles, Samuel 1998: Endogenous Preferences: The Cultural Consequences of Markets and Other Economic Institutions, in: Journal of Economic Literature 36: 1, 75-111.

Camerer, Colin F. 2003. Behavioral Game Theory: Experiments in Strategic Interaction, Princeton, NJ.

Camerer, Colin F./Fehr, Ernst 2006: When Does »Economic Man« Dominate Social Behavior?, in: Science 311, 47-52.

Checkel, Jeffrey T. 2001: Why Comply? Constructivism, Social Norms, and the Study of International Institutions, in: International Organization 55: 3, 553-588. 
Checkel, Jeffrey T. 2010: Theoretical Synthesis in IR: Possibilities and Limits, in: http:// www.sfu.ca/internationalstudies/PDFs/WP6.pdf; 04.03.2011.

Dana, Jason/Cain, Daylian M./Dawes, Robyn M. 2006: What You Don't Know Won't Hurt Me: Costly (but Quiet) Exit in Dictator Games, in: Organizational Behavior and Human Decision Processes 100: 2, 193-201.

Dana, Jason/Weber, Roberto A./Xi Kuang, Jason 2007: Exploiting Moral Wiggle Room: Experiments Demonstrating an Illusory Preference for Fairness, in: Economic Theory 33: 1, 67-80.

Deitelhoff, Nicole 2009: The Discursive Process of Legalization: Charting Islands of Persuasion in the ICC Case, in: International Organization 63: 1, 33-65.

Diekmann, Andreas/Eichner, Klaus/Schmidt, Peter/Voss, Thomas (Hrsg.) 2008: Rational Choice. Theoretische Analysen und empirische Resultate, Wiesbaden.

Dilthey, Wilhelm 1924: Die geistige Welt. Einleitung in die Philosophie des Lebens. Erste Hälfte: Abhandlungen zur Grundlegung der Geisteswissenschaften (Gesammelte Schriften, Band 5), Göttingen.

Elster, Jon 2007: Explaining Social Behavior: More Nuts and Bolts for the Social Sciences, Cambridge.

Esser, Hartmut 1993: Soziologie. Allgemeine Grundlagen, Frankfurt a. M.

Falk, Armin A./Heckman, James J. 2009: Lab Experiments Are a Major Source of Knowledge in the Social Sciences, in: Science 326, 535-538.

Fearon, James D./Wendt, Alexander 2003: Rationalism versus Constructivism: A Sceptical View, in: Carlsnaes, Walter/Risse, Thomas/Simmons, Beth A. (Hrsg.): Handbook of International Relations, London, 52-72.

Fehr, Ernst/Schmidt, Klaus 1999: A Theory of Fairness, Competition, and Cooperation, in: Quarterly Journal of Economics 114: 3, 817-868.

Forsythe, Robert/Horowitz, Joel L./Savin, Nathan E./Sefton, Martin 1994: Fairness in Simple Bargaining Experiments, in: Games and Economic Behavior 6: 3, 347-369.

Friedman, Jeffrey 1996: The Rational Choice Controversy: Economic Models of Politics Reconsidered, New Haven, CT.

Glazer, Jacob/Rubinstein, Ariel 2004: On Optimal Rules of Persuasion, in: Econometrica 72: 6, $1715-1736$.

Green, Donald P./Shapiro, Ian 1994: Pathologies of Rational Choice Theory: A Critique of Applications in Political Science, New Haven, CT.

Grobe, Christian 2009: Wie billig ist Reden wirklich? Kommunikative vs. strategische Rationalität in einem experimentellen Diktatorspiel, in: Zeitschrift für Internationale Beziehungen 16: 2, 269-297.

Grobe, Christian 2010: The Power of Words: Argumentative Persuasion in International Negotiations, in: European Journal of International Relations 16: 1, 5-29.

Güth, Werner/Kliemt, Hartmut/Peleg, Bezalel 2000: Co-evolution of Preferences and Information in Simple Games of Trust, in: German Economic Review 1: 1, 83-110.

Haisley, Emily C./Weber, Roberto A. 2010: Self-Serving Interpretations of Ambiguity in OtherRegarding Behavior, in: Games and Economic Behavior 68: 2, 614-625.

Hindmoor, Andrew 2010: Review Article: »Major Combat Operations Have Ended«? Arguing about Rational Choice, in: British Journal of Political Science 41: 1, 191-210.

HM Revenue \& Customs 2011: Webseite, in: http://www.hmrc.gov.uk/about/tax-defaulters-qa.htm; 29.07.2010.

Hollis, Martin 2002: The Philosophy of Social Science, 2. Auflage, Cambridge.

Katzenstein, Peter J./Keohane, Robert O./Krasner, Stephen D. 1998: International Organization and the Study of World Politics, in: International Organization 52: 4, 645-686.

Kennedy, Peter 2008: A Guide to Econometrics, 6. Auflage, Malden, MA.

Kirchgässner, Gebhard 2008: Homo Oeconomicus. Das ökonomische Modell individuellen Verhaltens und seine Anwendung in den Wirtschafts- und Sozialwissenschaften, 3. Auflage, Tübingen. 
Los Angeles Times 2011: Data Desk: California's Top Tax Scofflaws, in: http://projects.latimes.com/california/top-tax-scofflaws; 25.03.2011.

Marx, Johannes 2006: Vielfalt oder Einheit der Theorien in den Internationalen Beziehungen. Eine systematische Rekonstruktion, Integration und Bewertung, Baden-Baden.

Marx, Johannes 2010: Is There a Hard Core of IR? Eine wissenschaftstheoretische Betrachtung der Theorien der Internationalen Beziehungen, in: Zeitschrift für Internationale Beziehungen 17: 1, 41-74.

Morin, Jean-Frédéric/Gold, Richard E. 2010: Consensus-Seeking, Distrust and Rhetorical Entrapment: The WTO Decision on Access to Medicines, in: European Journal of International Relations 16: 4, 563-587.

Muller, Edward N./Opp, Karl-Dieter 1986: Rational Choice and Collective Action, in: American Political Science Review 80: 2, 471-487.

Müller, Harald 1995: Spielen hilft nicht immer. Die Grenzen des Rational-Choice-Ansatzes und der Platz der Theorie kommunikativen Handelns in der Analyse internationaler Beziehungen, in Zeitschrift für Internationale Beziehungen 2: 2, 371-391.

Olson, Mancur 1965: The Logic of Collective Action: Public Goods and the Theory of Groups, Cambridge, MA.

Opp, Karl-Dieter 1986: Soft Incentives and Collective Action: Participation in the Anti-NuclearMovement, British Journal of Political Science 16: 1, 87-112.

Opp, Karl-Dieter 1999: Contending Conceptions of the Theory of Rational Action, in: Journal of Theoretical Politics 11:2, 171-202.

Ostrom, Elinor 2006: The Value-Added of Laboratory Experiments for the Study of Institutions and Common-Pool Resources, in: Journal of Economic Behavior and Organization 61: 2, 149-163.

Plott, Charles R./Smith, Vernon L. (Hrsg.) 2008: Handbook of Experimental Economics Results, Amsterdam.

Riley, John G. 2001: Silver Signals: Twenty-Five Years of Screening and Signaling, in: Journal of Economic Literature 39: 2, 432-478.

Risse-Kappen, Thomas 1995: Reden ist nicht billig. Zur Debatte um Kommunikation und Rationalität, in: Zeitschrift für Internationale Beziehungen 2: 1, 171-184.

Risse, Thomas/Kleine, Mareike 2010: Deliberation in Negotiations, in: Journal of European Public Policy 17: 5, 708-726.

Sarigil, Zeki 2010: Bargaining in Institutionalized Settings: The Case of Turkish Reforms, in: European Journal of International Relations 16: 3, 463-483.

Schäfer, Gregor 2007: Spieltheorie und kommunikatives Handeln in den Internationalen Beziehungen. Eine Analyse der ZIB-Debatte (1994-2001), Marburg.

Vogt, Carsten 2000: The Evolution of Cooperation in Prisoner's Dilemma with an Endogenous Learning Mutant, in: Journal of Economic Behavior and Organization, 42: 3, 347-373.

Weber, Max 1972: Wirtschaft und Gesellschaft, 5. Auflage, Tübingen.

Wendt, Alexander 1999: Social Theory of International Politics, Cambridge. 